\title{
Robust measurements of electric conductivity in polyethylene based materials: measurement setup, data processing and impact of sample preparation
}

\author{
X.Xu ${ }^{1}$, M. Karlsson ${ }^{2}$, K. Gaska ${ }^{1}$, S. M. Gubanski ${ }^{1}$, H. Hillborg ${ }^{3}$, and U. W. Gedde ${ }^{2}$ \\ ${ }^{1}$ Materials and Manufacturing Technology, Chalmers University of Technology, \\ SE 412-96 Gothenburg, Sweden \\ ${ }^{2}$ Fibre and Polymer Technology, Royal Institute of Technology, SE-100 44 Stockholm, Sweden \\ ${ }^{3}$ ABB AB, Power Technology, Corporate Research, SE-721 78 Västerås, Sweden
}

\begin{abstract}
In this work, a measurement setup and an algorithm are introduced that allow for obtaining and processing the current data during standard measurements of electric conductivity in polymeric insulation materials. Robustness of this setup is demonstrated through dc conductivity measurements of low density polyethylene (LDPE) specimens that were prepared by two different techniques, i.e. compression molding and extrusion, at two independent laboratories (at Chalmers and KTH) from the same raw pellets. The results resented in this paper reveal that the implemented data processing algorithm allows for clear separation of the conduction current level and the external noise and thus for good differentiation between the conductivity levels in the investigated material specimens. In addition, a high reproducibility of the results is obtained for the independently manufactured specimens, suggesting the proposed measurement technique is well suitable for characterization of low conducting dielectrics.
\end{abstract}

\section{Introduction}

Electric conductivity of polyethylene based materials for applications in HVDC cable insulation belongs to the group of major design parameters [1]. Therefore its precise determination is required not only for understanding of the conduction mechanism nature but also for determining how material processing and sample preparation procedures influence this parameter 2].

The electric conductivity of LDPE based insulation materials is very low, often at the range of $10^{-16} \mathrm{~S} / \mathrm{m}[3$, which imposes a necessity to overcome severe metrological challenges during its characterization, as the measured current levels often reach the edge of modern electrometer's sensitivity limits [4]. The analysis of the measured current introduced in this paper allows for dynamically adjusting the average number of registered data points by evaluating the deviation of every incoming data. This not only allows to substantially increase the sensitivity of the measurement setup, but also to capture the faster current events. To verify the employed measurement technique, verification tests were performed independently at Chalmers (High Voltage
Engineering) and at Royal Institute of Technology (KTH), (Polymer Technology). Pressed and extruded low density polyethylene (LDPE) specimens were prepared for the carried dc conductivity measurements by both the labs. Visual inspections and scanning electron micrographs analyses clearly revealed structural differences in the prepared specimens while using the different preparation methods.

\section{Measurement setup}

A schematic view of the used dc conductivity measurement setups is shown in Figure 1. Keithley Electrometers (6517 series) are used to measure the current flowing through a specimen placed in a shielded electrode system. A high voltage DC supply (Glassman FJ60R2, 60kV) as well as the electrometer internal voltage supply (up to $1 \mathrm{kV}$ ) are utilized for securing a broad electric field range. A low pass filter (100 M $\Omega$ and $30 \mathrm{nF}$ ) for filtering out high frequency noise is integrated at the high voltage side. The advantage of this approach, as compared to a filter located after the test cell, allows registering by the electrometer any fast conduction process (faster than the time constant of the low pass filter) that may appear in the electrode-specimen setup. A software controlled overcurrent protection is introduced in the setup for switching off the voltage source when the current level exceeds a preset triggering level, for preventing possible electrometer damages in case of specimen breakdown. The used shielded electrode system is similar to a conventional three electrode system. The difference is that an additional shielding plate covers the back side of the measuring electrode for eliminating external capacitive couplings. To further

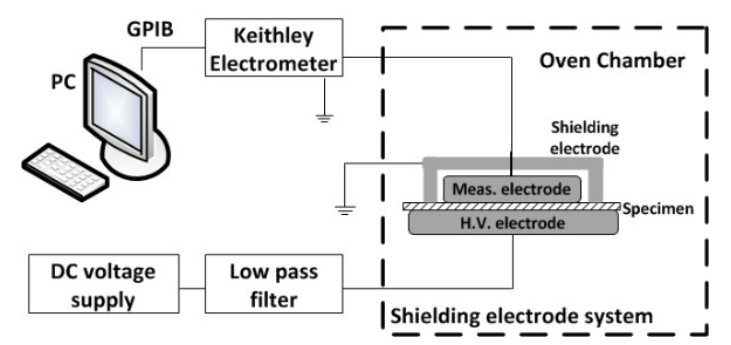

Fig. 1 - Schematic view of dc conductivity measurement setup. 
reduce influences by external noise, the whole electrode system is placed in a grounded stainless steel box. To control the temperature of the measurement, an oven is used. For stabilizing specimen temperature, the electrode system with inserted specimen are kept in the oven for at least 1 hour before each measurement.

\section{Data processing}

In dc conductivity measurement on modern polymeric insulation materials, an extensive averaging is often required for increasing the signal resolution. As illustrated in Figure 2, a typical current signal obtained from a LDPE specimen, 29 hours after energizing it. When the current level reaches sub-picoampere level, the data directly recorded from electrometer contain high amount of noise interferences, often even showing negative values. To increase the measurement resolution, varies averaging methods can be applied for obtaining a stable current signal. The mostly common ones are moving average and median filter. However, these may still not be able to provide high enough resolution for robust material characterization. As shown in the figure, results obtained by using different moving averages, median filters and even combination of the two methods still result in low signal to noise ratio. A more extensive data averaging is thus required and one possible approach is to dynamically adjusting the necessary averaging number, which allows suppressing the noise level while not hindering any fast conduction process that may appear during long time measurements. Another approach is to sample large amount of data points within a short time frame.

A LabVIEW based software has been developed for processing and recording the data in real-time. An algorithm, noted in the following as "smart average", is utilized to optimize the degree of necessary averaging by dynamically adapting to the signal to the noise level. The smart average function is realized by first evaluating the accumulated standard deviation (eq. 2.1) of every incoming data point.

$$
S_{n}=\sqrt{\frac{\left(a_{n}-A\right)^{2}+(n-1) S_{n-1}^{2}}{n}}
$$

where $\mathrm{A}$ is the previous recorded average data, and $a_{n}$ is the incoming data. Thereafter, by comparing the deviation of the recent incoming data points to the accumulated standard deviation, the algorithm decides on either changing the averaging number or on saving a record. If the deviation is larger than a certain pre-set value, the averaging number will be flushed to 1 . This functionality allows to capture any sudden significant changes in the measured current.

The results obtained by means of the smart average algorithm are also illustrated in Figure 2 and show that an identical measurement provides a much more stable result. The example in Figure 3 demonstrates the reduction of accumulated standard deviation with

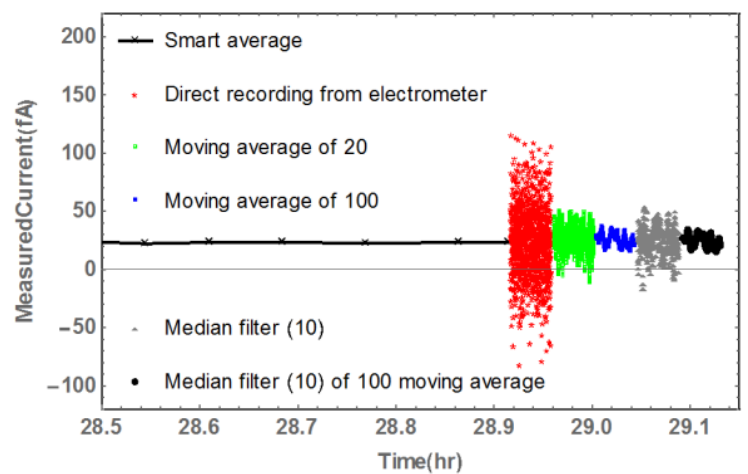

Fig. 2 - Illustration of the measured current using different averaging methods.

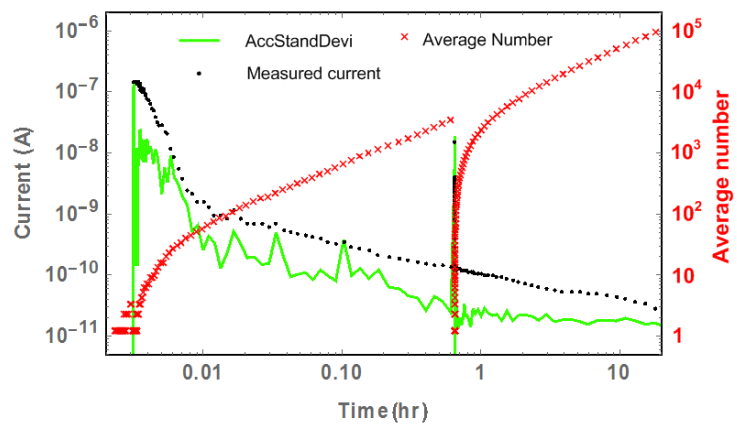

Fig. 3 - Accumulated standard deviation, used averaging number and measured current from dc conductivity measurement on LDPE specimen using smart average algorithm.

gradually decreasing measured current, thus increasing the average number. When a sudden change in the current appears (at around $30 \mathrm{~min}$ ), the algorithm triggers the flush of the average number and records a large current deviation, thereafter, the averaging number increases again with the following current decrease.

To maximize the sampling rate of the measurement setup, GPIB communication between electrometer and computer is realized, sampling rate of $10 \mathrm{~S} / \mathrm{s}$ is obtained with the highest data resolution $(6.5 \mathrm{~d})$ 4 from the electrometer.

As compared to direct recording, the smart average algorithm has a noticeable advantage in terms of recorded data size. For a 24 hours measurement at sampling rate of $10 \mathrm{~S} / \mathrm{s}$, the typical recoded data size by the smart average is less than 100 kilobyte. In contrast, it often reaches 50 megabyte data for the direct recording with the same amount of useful information. The difference in data size may not only limit the ability of post data processing but also challenges the ability of data logging software and memory of the computer during long time measurements that are required to obtain a stable current levels in polymeric dielectrics.

To verify the reliability of the smart average algorithm, Round robin test has been performed between Chalmers and KTH labs using different data processing methods and the obtained results show good agreement. One example is illustrated in Figure 4. Two specimens of the same material were separately measured by means of 


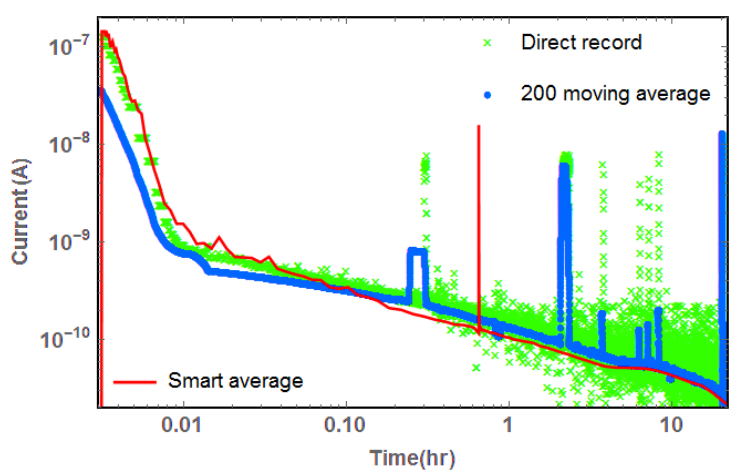

Fig. 4 - Comparison of smart average, direct record as well as 200 moving average on direct record.

smart average and direct recording, later the data from the direct recording were also post processed to obtain 200 moving average curve. As seen from the figure, in the first 5 minutes, when the current levels were well above the noise level, the results agree well between the smart averaging and the direct recording, but differ from the moving average result. This is because 200 average number is too high for clearing the large deviations in the measured current. However, when the current reduces below $10^{-10} \mathrm{~A}$, a good agreement can only be found between the smart average data and 200 moving average. It can thus be concluded that the smart average algorithm in combination with the developed measurement setup provides a robust technique in the measurements of $\mathrm{dc}$ conductivity of polymeric insulation materials.

\section{Materials specimens}

Plaque specimens for the study were independently made from LDPE pellets at Chalmers and KTH by pressing or extruding. The pressed specimen prepared in Chalmers were directly compression molded into $100 \mu \mathrm{m}$ thick films. The process started by increasing temperature of the mold to $130{ }^{\circ} \mathrm{C}$ at pressing force of $2 \mathrm{kN}$; thereafter, temperature was held at $130{ }^{\circ} \mathrm{C}$ for 3 minutes, allowing the pellets to melt, thereafter followed a gradual increase of the press force to $200 \mathrm{kN}$; finally the samples were cooled down to $20^{\circ} \mathrm{C}$ (by water cooling function of the press for 6 minutes) while keeping the press force at 200 $\mathrm{kN}$. Aluminum foil (ALU) and polyethylene terephthalate film (PET) were used as protective layers during the pressing.

The preparation of the extruded specimens at Chalmers was done with cryogenically grounded LDPE pellets into powder (average diameter of the particles $0.5 \mathrm{~mm}$ ), thereafter two extrusion cycles were used with a single screw Brabender extruder 19/25D. In the first extrusion, pellets of the master-batch were manufactured, which then were used in the second extrusion to obtain thin films with an average thickness of $100 \mu \mathrm{m}$. The extruder temperatures from the hopper to the die were respectively: $115,130,140$ and $140{ }^{\circ} \mathrm{C}$. A constant speed of $5 \mathrm{rpm}$ was kept for the compression screw 5 .

The pressed specimens were prepared at KTH by compression molding (LabPro 400 Press, Frontlijne

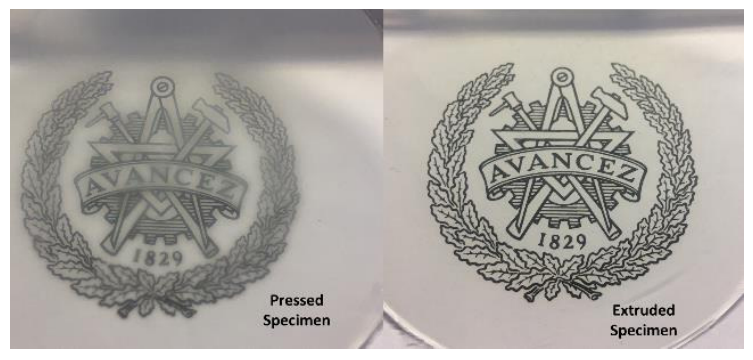

Fig. 5 - Image of the tested specimens prepared by KTH.

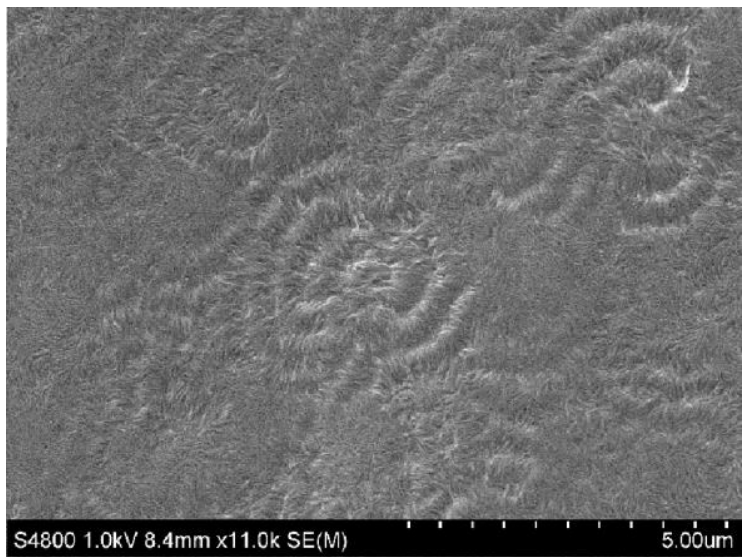

a) Chalmers pressed pellets

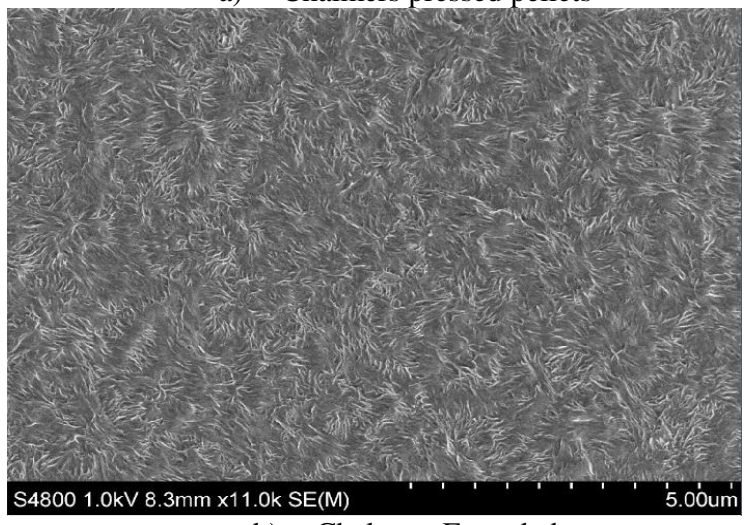

b) Chalmers Extruded

Fig. 6-Scanning electron micrographs of cross-sections from pressed or extruded specimens.

Grotnes) from LDPE pellets into $300 \mu \mathrm{m}$ thick film. It start with applying a contact pressure for 10 minutes at $130{ }^{\circ} \mathrm{C}$, followed by an additional 10 minutes pressing at $200 \mathrm{kN}$ and thereafter water cooling to room temperature for 7 minutes at the same press force of $200 \mathrm{kN}$. ALU foil and PET film were also used as protective material during compression molding.

The extrusion started at KTH by obtaining LDPE powder from cryo-grinding of pellets after immersion in liquid nitrogen for at least $1 \mathrm{~h}$. The powder was then circulated in an extruder (Micro 5cc Twin Screw Compounder, DSM Xplore) at $150{ }^{\circ} \mathrm{C}$ with speed of $100 \mathrm{rpm}$ for 6 minutes, thereafter, compression moulding was also used to obtain $300 \mu \mathrm{m}$ plaque specimen. Here only ALU foil was used as protective material.

Figure 5 shows images of two specimens from KTH, prepared by pressing and extrusion, indicating the degree of their transparency. The transparency of extruded 
specimen is clearly higher than that of pressed one, indicating existence of structural (morphological) differences. Similar effect was also found for the specimens prepared at Chalmers, though due to the thickness difference, these were not so clearly visible.

To further investigate the internal structural difference between the investigated specimens, scanning electron micrographs of their cross-section were analyzed on the materials prepared at Chalmers after freeze-cracking and acid etching of the amorphous domains. Figure 6 shows results obtained from pressed and extruded specimens. It can be seen that aggregates of banded spherulites were formed in the pressed specimens, while the extrusion often yielded a morphology without spherulites but rather well distributed axialites 6,7]. Further investigations on the impact of material morphology are continued.

\section{Results and discussion of de conductivity measurements}

DC conductivity of all the specimens was measured at electric field of $3.3 \mathrm{kV} / \mathrm{mm}$ and at temperature of $22^{\circ} \mathrm{C}$. Figure 7 shows the time dependence of the conductivity for the various specimens, based on the measured for 20 $\mathrm{h}$ current data and treated by means of the smart average algorithm. A difference, nearly of two orders of magnitude, is found between the extruded and pressed specimens, indicating the strong impact of material morphology 8]. The interface between crystalline and amorphous phases are believed to act as charge trapping sites, limiting the conduction currents flow [9]. For the pressed specimens, as their structure is more nonuniform, with domination of some amorphous areas, the resulting current becomes higher than in the extruded uniform structure. One may also notice a small influence brought up by the type of used protective material (ALU foil or PET films) during pressing, which can be caused by different surface roughness. Also small but consistent difference can be observed between the specimens manufactured by both the labs, which can be due to the difference in the used raw LDPE pellets. It is thus important pointing out that the presented results provide a good evidence of high resolution of the developed measurement technique.

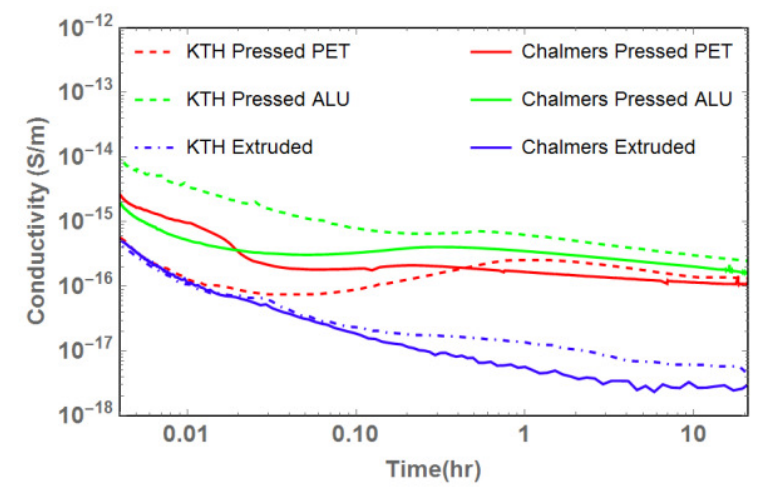

Fig. 7 - Conductivity of different specimens measured at $3.3 \mathrm{kV} / \mathrm{mm}$ and $22^{\circ} \mathrm{C}$.

\section{Acknowledgment}

This work has been carried out within a Swedish Research Council (VR - Vetenskapsrådet) project (E0539801). H. Hillborg also acknowledges the support from the Swedish Foundation for Strategic Research (SM14-0034).

\section{References}

[1] U. H. Nilsson, J. Andersson, V. Englund, V. Eriksson, P. O. Hagstrand, and A. Smedberg, "The role and measurement of DC conductivity for HVDC cable insulation materials," in 2015 IEEE Conference on Electrical Insulation and Dielectric Phenomena (CEIDP), 2015, pp. 3134.

[2] S. Boggs, D. H. Damon, J. Hjerrild, J. T. Holboll, and M. Henriksen, "Effect of insulation properties on the field grading of solid dielectric DC cable," IEEE Transactions on Power Delivery, vol. 16, pp. 456-461, 2001.

[3] M. G. Andersson, J. Hynynen, M. R. Andersson, V. Englund, P.-O. Hagstrand, T. Gkourmpis, et al., "Highly Insulating Polyethylene Blends for High-Voltage DirectCurrent Power Cables," ACS Macro Letters, vol. 6, pp. 78-82, 2017/02/21 2017.

[4] Keithley, "Keithley Electrometer Model 6517B Reference Manual," ed, 2016.

[5] K. Gaska, X. Xu, S. Gubanski, and R. Kádár, "Electrical, Mechanical, and Thermal Properties of LDPE Graphene Nanoplatelets Composites Produced by Means of Melt Extrusion Process," Polymers, vol. 9, p. 11, 2017.

[6] S. Murakami, S. Kohjiya, and K. Shimamura, "Comparative Study of Structure and Tensile Properties of Melt-Pressed and Extruded-Blown Films of High-Density Polyethylene," Journal of Macromolecular Science, Part B, vol. 39, pp. 645-655, 2000/11/27 2000.

[7] S. H. Han, Y. S. Yeom, J. G. Ko, H. C. Kang, and H. G. Yoon, "Effect of the degree of crystallinity on the electrical properties of MWCNT filled poly (ethylene-co-ethyl acrylate)/LDPE blend composites prepared by melt mixing," Composites Science and Technology, vol. 117, pp. 351-356, 9/29/2015. M. Ieda, "Electrical Conduction and Carrier Traps in Polymeric Materials," IEEE Transactions on Electrical Insulation, vol. EI19, pp. 162-178, 1984.

[9] G. Mazzanti and M. Marzinotto, Extruded Cables for High-Voltage Direct-Current Transmission: Advances in Research and Development: Wiley, 2013. 